\title{
Unmasked antegrade preexcitation of nodoventricular fiber following the slow pathway ablation: a case of successful mapping of the right ventricular insertion site
}

Gaku Izumi ${ }^{1}$, Hisashi Yokoshiki ${ }^{2}$ Ryo Sasaki ${ }^{1}$, Yuki Chiba ${ }^{1}$, Hirokuni Yamazawa ${ }^{1}$, and Atsuhito Takeda ${ }^{1}$

${ }^{1}$ Hokkaido University Graduate School of Medicine

${ }^{2}$ Sapporo City General Hospital

June 1, 2020

\begin{abstract}
We described a 15-year-old boy who underwent the catheter ablation for the nodoventricular tachycardia that had difficulty in differentiation from atrioventricular nodal reentrant tachycardia with upper common pathway. The slow pathway ablation revealed an anterograde conduction of nodoventricular fiber. We successfully performed the catheter ablation targeting for the right ventricular insertion site of the nodoventricular fiber.
\end{abstract}

\section{Hosted file}

manuscript for JCE(first).doc available at https://authorea.com/users/328578/articles/455792unmasked-antegrade-preexcitation-of-nodoventricular-fiber-following-the-slow-pathwayablation-a-case-of-successful-mapping-of-the-right-ventricular-insertion-site 
A B
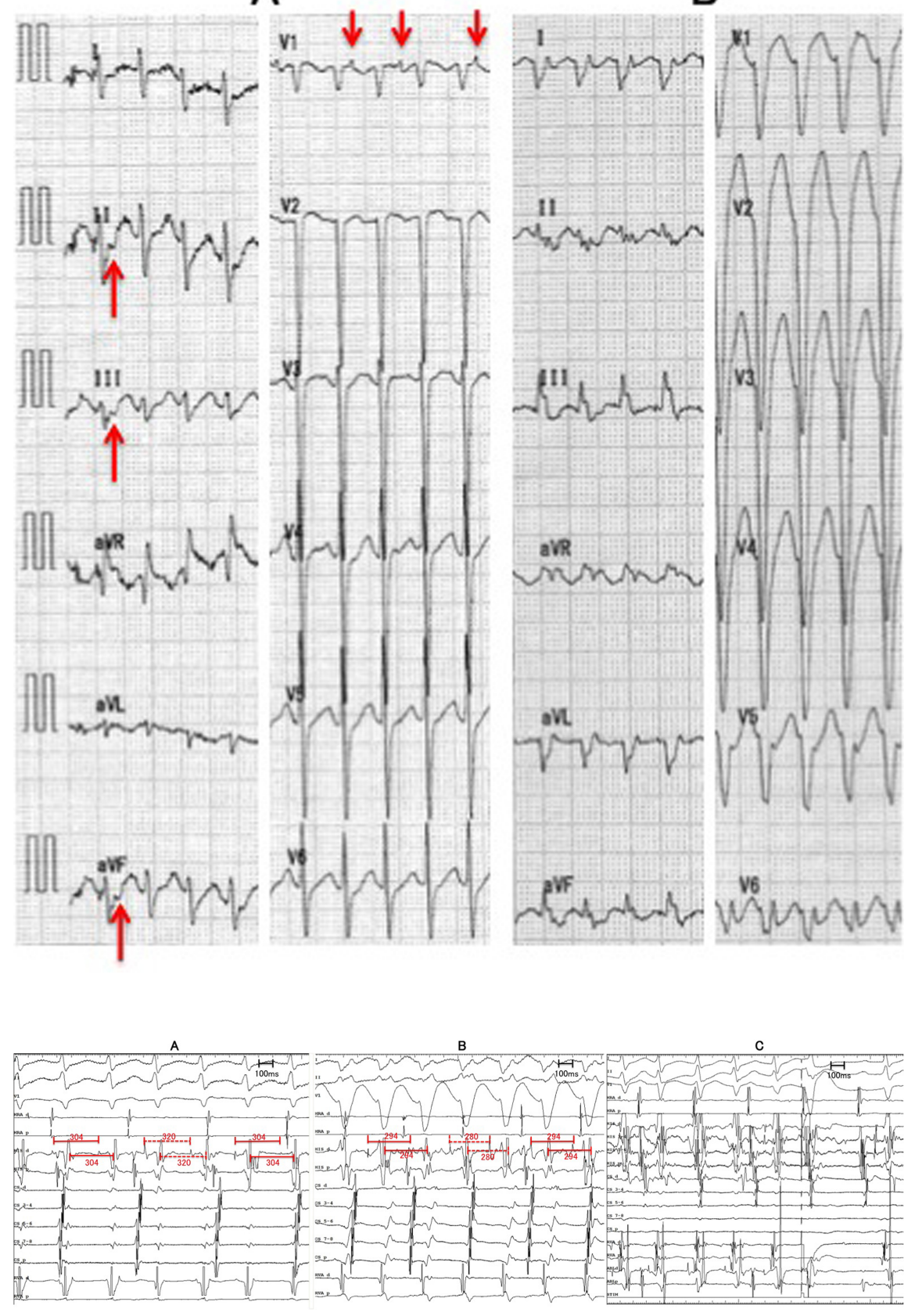


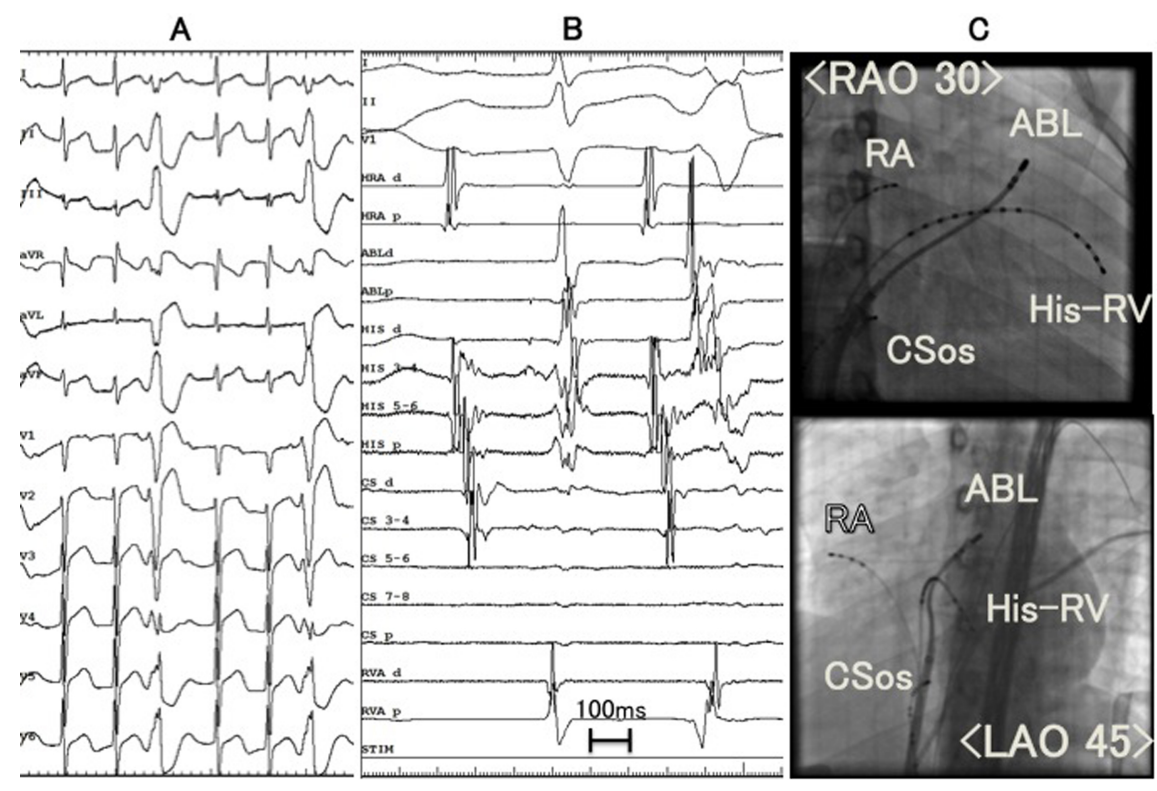

